CORRECTION

https://doi.org/10.1038/s41586-019-1133-3

\section{Author Correction: CPS1 maintains pyrimidine pools and DNA synthesis in KRAS/LKB1-mutant lung cancer cells}

Jiyeon Kim, Zeping Hu, Ling Cai, Kailong Li, Eunhee Choi, Brandon Faubert, Divya Bezwada, Jaime Rodriguez-Canales, Pamela Villalobos, Yu-Fen Lin, Min Ni, Kenneth E. Huffman, Luc Girard, Lauren A. Byers, Keziban Unsal-Kacmaz, Christopher G. Peña, John V. Heymach, Els Wauters, Johan Vansteenkiste, Diego H. Castrillon, Benjamin P. C. Chen, Ignacio Wistuba, Diether Lambrechts, Jian Xu, John D. Minna \& Ralph J. DeBerardinis

Correction to: Nature https://doi.org/10.1038/nature22359, published online 24 May 2017.

In this Letter, we used a multiple reaction monitoring (MRM) liquid chromatography-tandem mass spectrometry (LC-MS/MS) method to estimate relative quantities of carbamoyl phosphate in non-smallcell lung cancer (NSCLC) cell lines (Extended Data Fig. 1c). Further analysis has revealed that the MRM transition of 140/79 detects phosphorylethanolamine as well as carbamoyl phosphate. We improved the selectivity of the method by using more specific but less sensitive transitions (142/44 for phosphorylethanolamine and 140/97 for carbamoyl phosphate), and confirmed that the reported signal in Extended Data Fig. 1c was phosphorylethanolamine, not carbamoyl phosphate.
The method using these new transitions did not reproducibly detect carbamoyl phosphate, even in freshly prepared samples. To overcome the lability of carbamoyl phosphate in acidic conditions and increased temperatures ${ }^{1,2}$, we developed a derivatization method to transform it to a more stable 2-(2-pyridinyl)-hydrazinecarboxamide after a nucleophilic addition from 2-pyridylhydrazine. Although this method detects the intended derivative from a pure carbamoyl phosphate standard, the abundance of this product in NSCLC cell lines was below the background level. The low-level background of this derivatized product probably appeared through the slow, spontaneous conversion of 2-pyridylhydrazine to 2-(2-pyridinyl)-hydrazinecarboxamide under some conditions (for example, 2-pyridylhydrazine may react with cyanate ion at ambient temperature; see the formation of compound 66 in ref. ${ }^{3}$ ). We estimate the threshold for the detection of carbamoyl phosphate to be approximately 10 nanomoles, and we conclude that carbamoyl phosphate in our NSCLC extracts is below this threshold. We emphasize that these new data do not detract from the major findings of the paper: that LKB1 suppresses CPS1 expression in NSCLC, and that NSCLCs with concurrent mutations in KRAS and LKB1 require CPS1 to maintain pyrimidine pools. The original Letter has not been corrected online.

1. Lowenstein, J. M. \& Cohen, P. P. Studies on the biosynthesis of carbamylaspartic acid. J. Biol. Chem. 220, 57-70 (1956).

2. Wang, Q., Xia, J., Guallar, V., Krilov, G. \& Kantrowitz, E. R. Mechanism of thermal decomposition of carbamoyl phosphate and its stabilization by aspartate and ornithine transcarbamoylases. Proc. Natl Acad. Sci. USA 105, 16918-16923 (2008).

3. Haviv, F. et al. 3-[1-(2-Benzoxazolyl)hydrazino]propanenitrile derivatives: inhibitors of immune complex induced inflammation. J. Med. Chem. $\mathbf{3 1}$ 1719-1728 (1988). 